\title{
Efficiency Analysis of a Hall Thruster Operating with Krypton and Xenon
}

\author{
Jesse A. Linnell* and Alec D. Gallimore ${ }^{\dagger}$ \\ University of Michigan, Ann Arbor, Michigan 48109
}

DOI: $10.2514 / 1.19613$

\begin{abstract}
Krypton has recently become the focus of attention in the Hall thruster community because of its relatively large specific impulse compared with xenon and its potential to operate with comparable efficiencies. However, before krypton can be considered a viable propellant choice for missions, the performance gap between xenon and krypton must be reduced. A series of diagnostic measurements are taken for xenon and krypton propellant using the NASA-173Mv1 Hall thruster and the results are analyzed using a phenomenological performance model. The combined use of experiments and modeling enables a direct comparison of several efficiency components for each propellant to be made. With this method, it is possible to pinpoint the exact causes for the efficiency gap between xenon and krypton. It is also possible to see the effect of the magnetic field topology on Hall thruster performance and where gains are being made due to the magnetic field. Although there is a large series of competing components that differentiate krypton and xenon performance, the largest factors that dictate the efficiency difference between krypton and xenon are krypton's inferior propellant utilization and beam divergence. For xenon, the propellant utilization is $\mathbf{5 - 1 0 \%}$ higher and the beam divergence efficiency is approximately $\mathbf{8 \%}$ higher.
\end{abstract}

\begin{tabular}{ll} 
& \multicolumn{1}{c}{ Nomenclature } \\
$A_{c}$ & $=$ collector area \\
$B$ & $=$ magnetic flux density \\
$d$ & $=$ parallel plate gap distance \\
$E$ & $=$ electric field \\
$e$ & $=$ electron charge \\
$f(V)$ & $=$ ion voltage distribution function \\
$g(\theta)$ & $=$ normalized ion current density function at angle $\theta$ \\
$I_{b}$ & $=$ beam current \\
$I_{D}$ & $=$ discharge current \\
$I_{i}$ & $=$ current from $i$ th ion species \\
$I_{p}$ & $=$ probe current \\
$j(\theta)$ & $=$ ion current density at angle $\theta$ \\
$j_{\mathrm{FP}}(\theta)$ & $=$ ion current density measured by Faraday probe at \\
$M_{i}$ & $=$ angle $\theta$ \\
$\dot{m}_{a}$ & $=$ anode of propellant atom \\
$\dot{m}_{b}$ & $=$ total ion mass flow rate \\
$\dot{m}_{i}(\theta)$ & $=$ ion mass flow rate at angle $\theta$ \\
$n_{b}$ & $=$ total ion number density \\
$n_{i}$ & $=$ number density of $i$ th ion species \\
$P_{D}$ & $=$ discharge power \\
$V_{a}$ & $=$ acceleration voltage \\
$V_{a, \text { eff }}$ & $=$ effective acceleration voltage \\
$V_{a, i}$ & $=$ acceleration voltage of $i$ th species \\
$V_{D}$ & $=$ discharge voltage \\
$V_{l}$ & $=$ loss voltage \\
$V_{\mathrm{mp}}$ & $=$ most probable voltage \\
$V_{p}$ & $=$ plasma potential \\
$V_{\mathrm{probe}}$ & $=$ probe voltage \\
&
\end{tabular}

Presented at the 41st Joint Propulsion Conference, Tucson, FL, 10-11 July 2005; received 16 September 2005; revision received 25 January 2006; accepted for publication 19 February 2006. Copyright (C) 2006 by the American Institute of Aeronautics and Astronautics, Inc. All rights reserved. Copies of this paper may be made for personal or internal use, on condition that the copier pay the $\$ 10.00$ per-copy fee to the Copyright Clearance Center, Inc., 222 Rosewood Drive, Danvers, MA 01923; include the code $\$ 10.00$ in correspondence with the CCC.

${ }^{*}$ Ph.D. Candidate, Department of Aerospace Engineering, Plasmadynamics and Electric Propulsion Laboratory, 1919 Green Road, Room B107; jlinnell@umich.edu. Member AIAA.

${ }^{\dagger}$ Professor and Laboratory Director, Department of Aerospace Engineering, Plasmadynamics and Electric Propulsion Laboratory; alec.gallimore@ umich.edu. Associate Fellow AIAA.

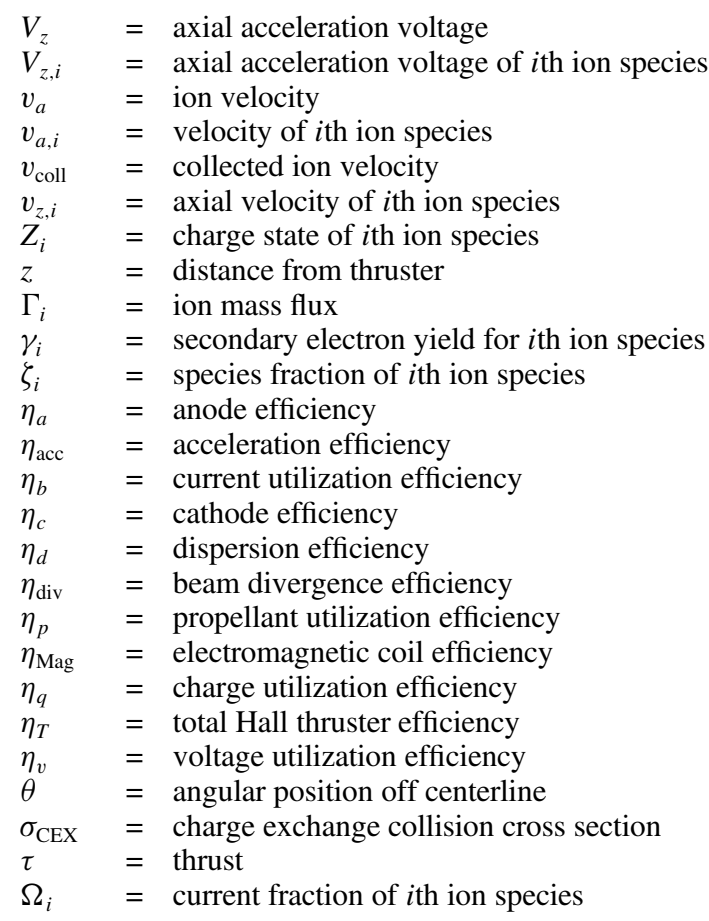

\section{Introduction}

$\mathbf{H}$ ALL thrusters [1,2] are space propulsion devices that use crossed electric and magnetic fields to ionize and accelerate propellant atoms to high exhaust velocities. A self-consistent electric field is established in the electron flow, which is impeded by an externally applied magnetic field. The magnetic and electric fields cause the electrons to follow a closed drift path, and for this reason Hall thrusters are often referred to as closed drift thrusters. Generally, noble gases of high atomic weight are used as propellant.

Of the noble gases, xenon has historically been the preferred propellant because of its high molecular weight and low ionization potential. The use of a lighter propellant increases ion velocities and therefore can increase specific impulse that in turn extends Hall thrusters into a larger range of mission applications. However, the higher ionization potentials of the lighter noble gases result in an efficiency deficit that has precluded these propellants from any 
serious discussion as viable options for space application. Although previous studies [3-7] report krypton to have an inferior performance compared with xenon, results using the NASA-457M [8,9] and the NASA-400M [10] indicate that krypton can be operated at efficiencies comparable to xenon. Because of the relative high price and scarcity of xenon and the superior specific impulse of krypton, krypton has recently sparked interest in the electric propulsion community. Before krypton can become a legitimate option for space propulsion, the reasons for the krypton efficiency gap must be studied and addressed. This is the motivation for the detailed efficiency study presented in this paper.

This paper presents a series of experimental results using the NASA-173Mv1 Hall thruster. The diagnostics used include a retarding potential analyzer (RPA), $E \times B$ probe, cylindrical Langmuir probe, and Faraday probe. These measurements are then applied to a performance model presented by Hofer [11] to isolate the efficiency differences between krypton and xenon. The efficiency analysis separates the anode efficiency into separate components, which allows one to evaluate the specific performance differences between krypton and xenon, which helps to define a direction for improving krypton efficiency in future thrusters.

\section{Experimental Apparatus and Techniques}

\section{A. Facility}

The measurements reported in this paper were conducted in the Large Vacuum Test Facility (LVTF) at the University of Michigan's Plasmadynamics and Electric Propulsion Laboratory (PEPL). The LVTF is a cylindrical stainless-steel tank that is $9 \mathrm{~m}$ long and $6 \mathrm{~m}$ in diameter. The vacuum chamber is evacuated using seven CVI model TM-1200 internal cryopumps. The pumps are capable of pumping $240,000 \mathrm{l} / \mathrm{s}$ of xenon and 252, $000 \mathrm{l} / \mathrm{s}$ of krypton. The pressure is monitored by using two hot-cathode ionization gauges. The vacuum chamber operates at a base pressure of $1.5 \times 10^{-7}$ torr and approximately $3.2 \times 10^{-6}$ torr (corrected [12]) during both the krypton and xenon thruster operation points.

High-purity research grade xenon and krypton are used as propellants for the following measurements. The purity level of xenon and krypton are $99.9995 \%$ and $99.999 \%$, respectively. The propellants are supplied through propellant feel lines using 20 and $200 \mathrm{sccm}$ mass flow controllers for the cathode and anode, respectively. The mass flow controllers are calibrated using a constant volume method. The compressibility correction factor for xenon and krypton are calculated using the van der Waals equation [13] and the Virial equation [14]. Error in the mass flow controllers is approximately $\pm 1 \%$ of full scale.

\section{B. Thruster}

The NASA-173Mv1 Hall effect thruster (Fig. 1) $[11,15]$ is used in the following experiment. In addition to the standard inner and outer magnetic coils, the NASA-173Mv1 uses a trim coil to create a highly adaptable magnetic field topology. The added magnetic field control offered by the trim coil is found to improve thruster efficiency through plasma lens focusing and a magnetic mirroring effect that focus the electrons and ions toward the center of the discharge channel. This topology has been shown to improve thruster efficiency by improving beam focusing and the electron dynamics [11].

A Busek BHC-50-3UM cathode is used for this experiment. For all of the thruster operation points, the cathode flow rate is equal to $10 \%$ of the anode flow rate with a minimum flow rate of $0.93 \mathrm{sccm}$. The cathode axial centerline is mounted $30 \mathrm{deg}$ off the axial thruster direction and the center of the cathode orifice is placed $30 \mathrm{~mm}$ downstream and $30 \mathrm{~mm}$ above the thruster face.

\section{Performance Diagnostics}

The thrust measurements for this experiment are recorded using a null-type inverted pendulum thrust stand, the same thrust stand design used with the NASA-457M [8,9]. For the performance measurements, thruster operation is monitored in real time by an

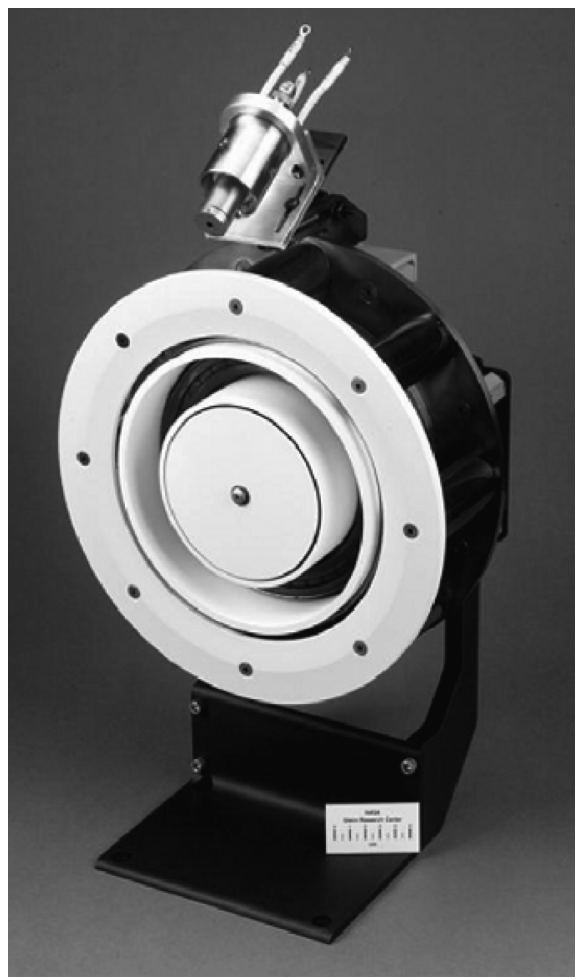

Fig. 1 NASA-173Mv1 Hall thruster.

Agilent data logger. The monitored properties include the magnet currents and voltages, discharge current, and thrust. The mass flow rate and discharge voltage are kept constant during the thruster tuning and therefore are inputted manually. Other than the discharge current, which is monitored via a current probe, currents are monitored by measuring the voltages across calibrated shunts. The magnet voltages are measured directly by the data logger. Thrust is measured by monitoring the thrust stand outputs and converting the voltage to thrust by a calibration curve. The optimal efficiency is found for each operation point by monitoring Hall thruster conditions and thrust efficiency in real time. As the magnet currents are altered, the efficiency adjusts in response to the changing magnetic field and the peak efficiency is attained. For most of the reported data, the thruster is allowed to run for two $h$ to find the true optimized performance settings. No operation points are run for less than 30 min.

The data logger inputs are calibrated using a digital multimeter. The error associated with the DC voltage measurements are $\pm 0.4 \%$ and $\pm 1.5 \%$ for DC current measurements. The current probe has approximately $\pm 1.5 \%$ error. Thrust, anode specific impulse, and anode efficiency measurement uncertainties are found by accounting for all aforementioned errors. Thrust measurements have $\pm 4.13 \mathrm{mN}$ error, anode specific impulse measurements have approximately $\pm 2.5 \%$ error, and anode efficiency measurements have a $5 \%$ relative error on average [16].

\section{Retarding Potential Analyzer}

The retarding potential analyzer [17-19] diagnostic uses a series of grids to determine the ion energy distribution by selectively filtering ions on the basis of kinetic energy. The first grid is floating, to minimize the perturbation of the sampled plasma. The second grid is negatively biased $(-30 \mathrm{~V})$, to repel all plasma electrons effectively. The third grid is used to retard the ions, so that only ions with energy-to-charge ratios greater than the grid voltage can pass through the retarding grid and reach the collector. The voltage of the retarding grid can be varied to determine the current-voltage characteristic. The derivative of the current-voltage characteristic is proportional to the ion energy distribution and can be seen in the idealized Eq. (1). It should be noted that because the RPA acts as a filter with a characteristic transfer function, it must be calibrated [20]. 


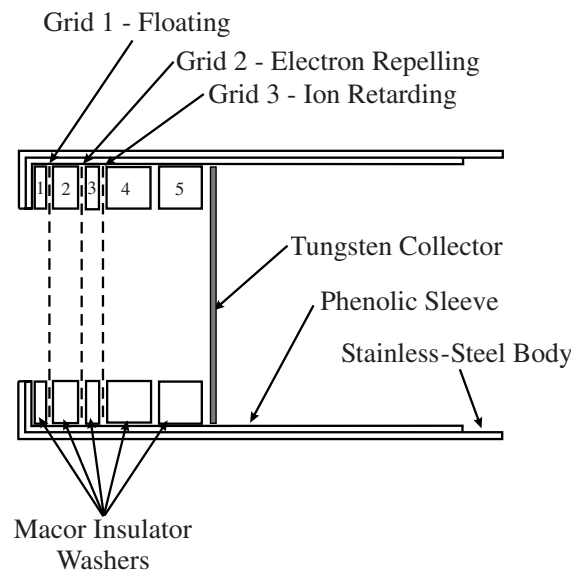

Fig. 2 Retarding potential analyzer schematic.

The schematic of the three-grid design appears in Fig. 2, and the potential diagrams of the RPA can be seen in Fig. 3 .

$$
d I_{p} / d V_{\text {probe }}=\frac{-Z_{i}^{2} e^{2} n_{b} A_{c}}{M_{i}} f(V)
$$

The outer body of the RPA is constructed of 316 stainless steel, which is held at ground potential. A phenolic sleeve placed inside the body provides electrical isolation of the grids. All grids are identical and are cut from 316 stainless steel, photochemically machined sheets with a thickness of $0.127 \mathrm{~mm}$ (0.005 in.). The grid openings are $0.2794 \mathrm{~mm}(0.011 \mathrm{in}$.) in diameter and the grid has an open area fraction of $38 \%$. Grid spacing is achieved using Macor washers machined to provide the correct separation. The collector is a tungsten-coated stainless-steel disc. A tungsten coating is used to reduce secondary electron emission from the collector. Electrical connections are accomplished by spot-welding stainless-steel wire to each grid. The wires are then routed along the inner edge of the phenolic sleeve and out the rear of the RPA body. The washers and grids are compressed by a spring placed behind the collector and held in place by a rear cover.

During this investigation, RPA data are taken $1 \mathrm{~m}$ downstream of the thruster exit plane from 30 to 90 deg off centerline in 15-deg increments. Inside of the 30-deg cone, the plasma density is often too high for proper RPA operation. RPA data as close as 20 deg off centerline are possible for the operation points with lower discharge currents. Data collection on centerline is exceedingly difficult and attempts at data collection on axis resulted in complete RPA failure. It is shown in Sec. IV.B that inside the cone angle of approximately $45 \mathrm{deg}$, the most probable ion voltage is constant. Therefore, the lack of RPA measurements inside the 30-deg cone is not seen as an issue for this investigation.

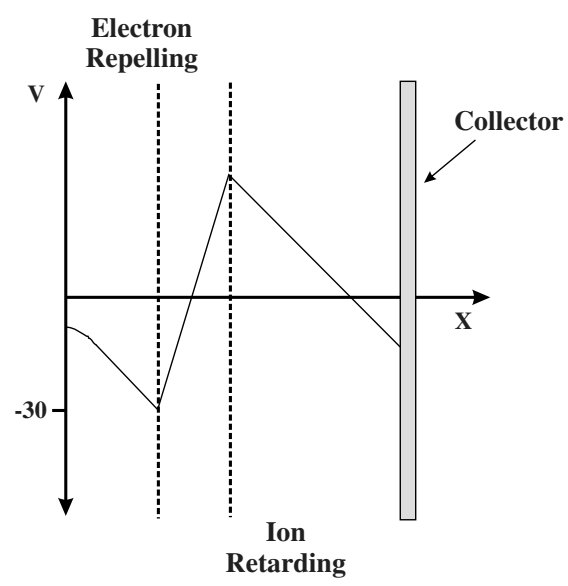

Fig. 3 Retarding potential analyzed potential diagram.
The RPA is used to provide estimates of the average ion acceleration kinetic energy. Because of possible difficulty in integrating the energy distribution function due to probe noise, the most probable voltage is used as an estimate of the average ion kinetic energy in electron volts. Because the ion-retarding grid applies a voltage with respect to facility ground, it is necessary to correct the ion energy-per-charge distribution function for the plasma potential $\left(V_{a}=V_{\mathrm{mp}}-V_{p}\right)$. The plasma potential is measured using a Langmuir probe positioned at $1 \mathrm{~m}$ downstream at $30 \mathrm{deg}$ off centerline. The Langmuir probe measurements are conducted using ESPion from Hiden Analytical. The Langmuir probe is cylindrical in shape with a diameter and length of 0.1 and $15 \mathrm{~mm}$, respectively. The orbital motion limited assumption is used to analyze the Langmuir probe data. The same plasma potential correction is used for all RPA positions from 90 to $20 \mathrm{deg}$ off centerline. Gulczinski [21] found almost no plasma potential variation with angular position in the similar UM/AFRL P5 Hall thruster and Walker's [22] work with the P5 found plasma potential variation of no more than $4 \mathrm{~V}$ between 20 and 90 deg off centerline. The sources of error associated with the RPA result in an uncertainty in the most probable voltage of $\pm 10 \mathrm{~V}$ [11].

The current collected by the RPA is processed by using a smoothing spline [23] to reduce the signal noise. As shown in Eq. (1), the normalized ion energy-per-charge distribution function is found by taking the derivative of the current with respect to voltage using a central difference method. The collected current, spline smoothed collected current, and the resulting ion voltage distribution function (VDF) are shown in Fig. 4.

\section{E. Wien Filter $E \times B$ Probe}

An $E \times B$ probe, otherwise known as a Wien filter, is a commonly used tool for measuring ion species fractions $[11,12,24,25]$. The $E \times B$ probe uses the Lorentz force to select ions of a specific velocity for collection. This filtering is accomplished through crossed electric and magnetic fields that are mutually perpendicular to the ion velocity vector. Generally, a constant magnetic field is provided along with an applied variable potential between two parallel plates to create perpendicular fields. For a particular ion velocity [see Eq. (2)], the Lorentz force vanishes and those ions can be collected by the probe. The $E \times B$ probe acts purely as a velocity filter and the collected ions are independent of mass and charge. While the $E \times B$ probe will not detect signatures due to charge exchange (CEX) collisions, ions that have undergone elastic collisions will cause a broadening of the $E \times B$ probe traces.

$$
v_{\mathrm{coll}}=E / B
$$

The ion charge state can be determined by considering the relationship between ion energy-per-charge and charge state to the applied plate voltage. When the ion velocity [Eq. (3)] is substituted into Eq. (2), by solving for the plate voltage, one arrives at the relation given in Eq. (4) in terms of acceleration potential and charge state.

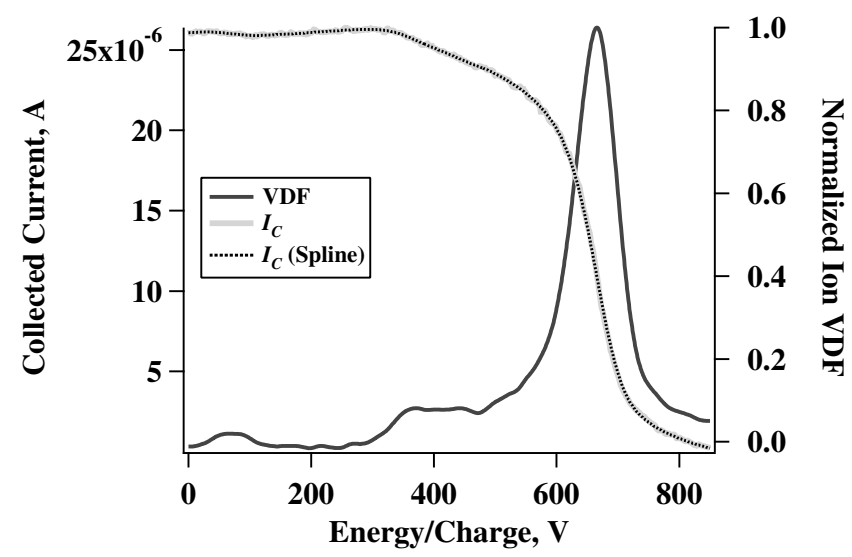

Fig. 4 Retarding potential analyzer data processing example. 
Because the acceleration potential of all ion species is approximately equal [25] (refer to Sec. III.B for further discussion), multiply charged species peaks will appear approximately at $Z_{i}^{1 / 2}$ times the probe voltage of the singly charged peak.

$$
\begin{gathered}
v_{a, i}=\sqrt{2 Z_{i} e V_{a, i} / M_{i}} \\
V_{\text {probe }}=\sqrt{2 Z_{i} e V_{a, i} / M_{i}}(B d)
\end{gathered}
$$

The $E \times B$ probe used in this study was originally designed, built, and calibrated by Kim [25]. The $E \times B$ test section is $254 \mathrm{~mm}$ long. The magnetic field supplied by four ceramic permanent magnets in the $E \times B$ test section averages $0.162 \mathrm{~T}$ and the variation along the length of the device is less than $10 \%$ [25]. The electric field is applied between two rectangular aluminum electrodes separated by a distance $d$ of $1.90 \mathrm{~cm}$. The entrance collimator is $152 \mathrm{~mm}$ in length and uses an entrance orifice of $1.5 \mathrm{~mm}$ in diameter. The exit collimator is $152 \mathrm{~mm}$ long and is connected to a 23-mm-diam tungsten collector.

The dimensions of the $E \times B$ probe are identical to previous experiments $[22,24]$ with the exception of the entrance orifice, which is added to reduce the collected signal. Based on these dimensions, the half-cone acceptance angle is estimated to be $0.56 \mathrm{deg}$ and the probe resolution is estimated to be approximately $7 \%$ of the ion energy [25]. The $E \times B$ test section is located $1.5 \mathrm{~m}$ downstream on the thruster centerline. The entrance and exit collimators are aligned perpendicularly to the thruster exit plane.

The electrodes in the $E \times B$ probe are biased at equal voltages above and below ground by a Keithley SourceMeter®. A picoammeter records the current to the plate, which is given by Eq. (5). For the ion energies reported in this experiment, the secondary electron yield of tungsten is $0.058,0.28,0.78$, and 1.75 for $\mathrm{Kr}^{+}, \mathrm{Kr}^{2+}, \mathrm{Kr}^{3+}$, and $\mathrm{Kr}^{4+}$, respectively, and $0.018,0.18,0.69$, and 1.46 for $\mathrm{Xe}^{+}, \mathrm{Xe}^{2+}, \mathrm{Xe}^{3+}$, and $\mathrm{Xe}^{4+}$, respectively [26].

$$
I_{i}=e Z_{i} n_{i} v_{a, i} A_{c}\left(1+\gamma_{i}\right)
$$

From the ion currents, the current fractions [Eq. (6)] and species fractions [Eq. (7)] are calculated. These values will be used in the analysis in the following sections.

$$
\begin{aligned}
& \Omega_{i}=I_{i} / \sum I_{i} \\
& \zeta_{i}=n_{i} / \sum n_{i}
\end{aligned}
$$

Equations (3) and (5) are inserted into Eq. (7) to solve the species fraction. Equations (3), (5), and (7) are then inserted into Eq. (6) to arrive at a new equation for ion current fractions in Eq. (8).

$$
\Omega_{i}=\frac{Z_{i}^{3 / 2} \zeta_{i}\left(1+\gamma_{i}\right)}{\sum Z_{i}^{3 / 2} \zeta_{i}\left(1+\gamma_{i}\right)}
$$

The method used to measure the area under the separate species peaks is described next. First an ensemble average of three separate voltage sweeps is taken. The averaged data are then smoothed using a smoothing spline to reduce noise. Starting with the highest charge state, a Gaussian curve fit is matched to the data and then the curve fit is subtracted from the lower charge-state species peaks. The process is then continued with the next highest charge state and repeated until all charge states have been analyzed. The current is subtracted to avoid double-counting the collected current. The process begins with the highest charge state to avoid problems that can occur due to poor curve fits. Effort is taken to use only sections of the species peaks that are far enough away from neighboring peaks so that the curve fits will not be affected by neighboring species peaks. An example of this method is shown in Fig. 5. In this figure, the solid black lines represent the Gaussian curve fits, the dotted black line is the summed curve fits, and the collected current from the $E \times B$ probe is given by

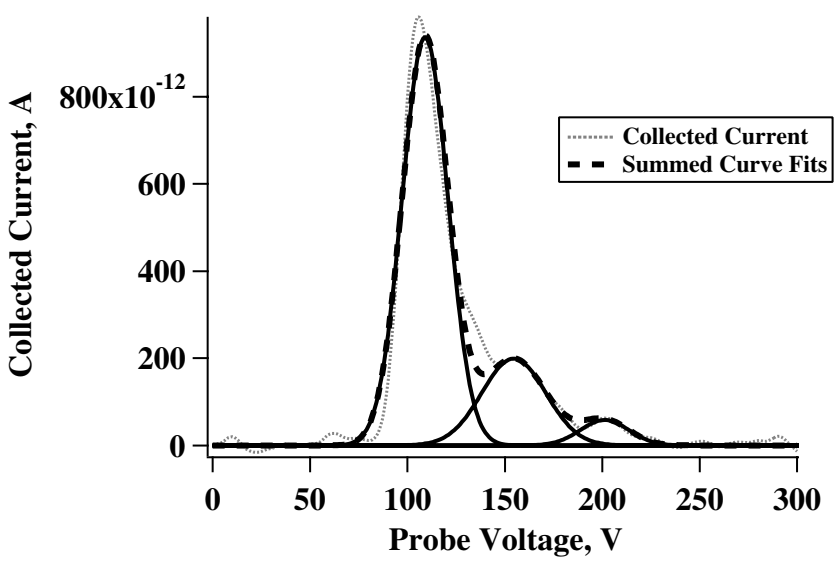

Fig. $5 E \times B$ ion current integration method.

the gray line. When this method is compared with methods used in previous work [24], there is no significant difference in the species fraction calculations.

The error in the species fraction calculation is approximately 4, 25 , 50 , and $150 \%$ for $\mathrm{Xe}^{+} / \mathrm{Kr}^{+}, \mathrm{Xe}^{2+} / \mathrm{Kr}^{2+}, \mathrm{Xe}^{3+} / \mathrm{Kr}^{3+}$, and $\mathrm{Xe}^{4+}$, respectively. These errors stem from a combination of voltage and current measurement inaccuracy, probe misalignment, probe resolution, loss of ions due to CEX collisions, and variation of ion species velocity. The effect of particle buildup and collision inside the probe was addressed by Kim [25] and found to be small compared with other experimental errors.

\section{F. Magnetically Filtered Faraday Probe}

Faraday probe data are collected using a magnetically filtered Faraday probe (MFFP) $[22,27,28]$. Facility effects and high back pressure can result in inaccurate Faraday probe measurements. The predominant effect of high back pressure that manifests itself as artificially large current measurements is the charge exchange (CEX) ion. The CEX ion is produced when a "fast" ion interacts with a "slow" neutral by exchanging an electron. The result is a fast neutral and slow ion. Low-energy ions are drawn to the negatively biased collector and the Faraday probe is unable to distinguish the difference between the fast and slow ions. Because of this, standard current density measurements tend to overpredict the ion beam current. At large angles off centerline, the measured current is largely CEX ions. The magnetically filtered Faraday probe has been shown to be very effective at excluding CEX ions. For this reason, the MFFP is chosen for the following analysis.

The MFFP has a collector surrounded by a box with a magnetic field applied inside the box. The magnetic field alters the trajectory of ions such that ions with kinetic energies below $20 \mathrm{eV}$ are deflected away from the collector. In addition, the box surrounding the collector acts as a geometric collimator that further reduces CEX ion collection. The collimator acts to reduce the effective collection area of the probe [29] and the reported results are corrected accordingly. Thus, the box and magnetic field result in a dual-mode ion filtration system.

Because of the large inaccuracy in Faraday probe measurements, certain sources of error must be addressed. Secondary electron emission occurs when high-energy ions collide with the collector and a low-energy electron is released from the surface. Assuming that the plasma is predominantly singly charged, the use of a tungsten collector greatly reduces the effect of secondary electron emission and this effect can be considered negligible $[26,30]$.

Another source of error connected to facility backpressure and high neutral density in the plume is plume attenuation. At high neutral densities, fewer ions are capable of reaching the collector without suffering a CEX collision. Attenuation is the decrease in beam current due to these collisions. By considering the onedimensional ion continuity equation and integrating over the path length $z$, one will arrive at the attenuation correction [31] in Eq. (9). The collector takes angular sweeps and is mounted $1 \mathrm{~m}$ downstream 
of the thruster. The operation pressure of the facility is approximately $3.2 \times 10^{-6}$ torr and a neutral temperature of $300 \mathrm{~K}$ is assumed. The CEX collision cross sections for xenon and krypton are approximately 51 and $40 B R O K E N^{2}$, respectively [32,33].

$$
j(\theta) / j_{\mathrm{FP}}(\theta)=\exp \left(n_{b} \sigma_{\mathrm{CEX}} z\right)
$$

The beam current is calculated from the Faraday probe data by integrating from 0 to $90 \mathrm{deg}$ in spherical coordinates [34]. The normalized current density function can be calculated by dividing the current density by the beam current. As is necessary with any filtering diagnostic with a characteristic transfer function, the MFFP has been calibrated $[27,28]$.

\section{Performance Analysis}

\section{A. Phenomenological Performance Model}

A phenomenological performance model developed by Hofer [11] separates the Hall thruster anode efficiency into four separate terms: the charge utilization, the propellant utilization, the current utilization, and the voltage utilization. With the efficiencies separated, it is possible to create distinctions in the importance of the individual efficiencies. In the case of krypton operation, it should be possible to pinpoint the different features that lead to the efficiency gap.

Total Hall thruster efficiency is a combination of anode efficiency, cathode efficiency, and electromagnetic coil efficiency. The anode efficiency can be further broken down into the four aforementioned partial efficiencies. Equation (10) shows the total efficiency, whereas Eq. (11) gives the equation for anode efficiency.

$$
\begin{gathered}
\eta_{T}=\eta_{c} \eta_{\mathrm{Mag}} \eta_{a} \\
\eta_{a}=\tau^{2} /\left(2 \dot{m}_{a} P_{D}\right)=\eta_{b} \eta_{p} \eta_{q} \eta_{v}
\end{gathered}
$$

The partial efficiencies are defined as follows. Current utilization efficiency is the amount of ion current compared with the discharge current and is given in Eq. (12). Voltage utilization efficiency is the measure of the amount of the discharge voltage (potential energy) that is converted into axial ion kinetic energy and is defined in Eq. (13). Propellant utilization is the amount of neutral anode flow that is converting into ion flow and is given in Eq. (14). Finally, charge utilization is the measure of the overall charge state of the beam ions [Eq. (15)]. Based on a series of diagnostic measurements, values for these separate efficiencies can be calculated.

$$
\begin{gathered}
\eta_{b}=I_{b} / I_{d} \\
\eta_{v}=V_{a, \text { eff }} / V_{D}=1-V_{l} / V_{D} \\
\eta_{p}=\frac{\dot{m}_{i}}{\dot{m}_{a}}=\frac{M_{i} I_{D}}{\dot{m}_{a} e} \eta_{b} \sum \frac{\Omega_{i}}{Z_{i}} \\
\eta_{q}=\left(\sum \frac{\Omega_{i}}{\sqrt{Z_{i}}}\right)^{2} / \sum \frac{\Omega_{i}}{Z_{i}}
\end{gathered}
$$

Based on probe measurement uncertainty, the charge utilization efficiency has a relative error of less than $1 \%$ for krypton and less than $2 \%$ for xenon. The propellant utilization efficiency and current utilization efficiency can be calculated based on the diagnostic measurements and the other efficiency calculations. The relative errors are 3.1 and $4.4 \%$ for current utilization and propellant utilization efficiency, respectively (for both xenon and krypton). Voltage utilization efficiency error will be covered in the next section.

\section{B. Acceleration and Beam Divergence Efficiency}

The assumptions used in the derivation of acceleration and beam divergence efficiency are summarized next. These assumptions are covered in greater detail in the text.

1) The average acceleration voltage is equal for all ion species.

2) The average acceleration voltage is constant at all angular positions in the beam.

3) The species fractions along the centerline are a good representation of the species fractions in the entire thruster plume.

4) The species fractions are approximately constant at different angular positions in the plume.

While the Hofer performance model is extremely useful for conducting a detailed study of Hall thruster performance as it is, a slight modification can be applied to measure the voltage utilization efficiency more accurately. The problem in the existing method of measuring the voltage utilization is not a flaw in the theory, but in the application of the theory. Previously [17], the voltage utilization has been calculated by taking an RPA measurement to calculate the average ion kinetic energy. While being able to capture the average kinetic energy of the ions, RPA measurements are incapable of capturing the entire voltage utilization efficiency.

Voltage utilization efficiency is a measurement of the effective axially directed ion kinetic energy in electron volts compared with the thruster discharge voltage. The energy loss is mainly a combination of spread in the energy-per-charge distribution function (dispersion efficiency), failure of the plasma to drop to cathode potential, and radial beam divergence. The energy loss is also affected by the ionization potential of the propellant, wall losses, and ion charge state. The correct calculation of the average axial ion energy requires a mass-weighted average of the ion energy over the entire angular range of the thruster plume [see Eq. (16)]. Furthermore, this analysis should take into account the effects of multiply charged species. Voltage utilization can be broken into two measurable components: acceleration efficiency and beam divergence efficiency. The acceleration efficiency is a way of quantifying the average ion kinetic energy and the beam divergence efficiency is a measure of the divergence loss in the beam. Hofer's voltage utilization efficiency [given in Eq. (13)] is broken into the acceleration and divergence components in Eqs. (17) and (18), respectively.

$$
\begin{gathered}
\eta_{v}=\eta_{\mathrm{acc}} \eta_{\mathrm{div}}=\frac{\left\langle\dot{m}_{i}(\theta) V_{z}(\theta)\right\rangle}{\dot{m}_{b} V_{D}} \\
\eta_{\mathrm{acc}}=V_{a} / V_{D} \\
\eta_{\mathrm{div}}=\frac{\left\langle\dot{m}_{i}(\theta) V_{z}(\theta)\right\rangle}{\dot{m}_{b} V_{a}}
\end{gathered}
$$

Using a combination of RPA, Faraday probe, and $E \times B$ measurements, a more rigorous analysis of the voltage utilization efficiency can be conducted. This study shows that the beam divergence efficiency is between approximately $75-90 \%$ for xenon and krypton. Previous studies that neglected the beam divergence efficiency [17] have underpredicted the current and propellant utilizations by approximately $5-15 \%$. Note that effort has been made to be consistent with Hofer's original model, and the original model should be referenced as a guide to some of the finer details in this derivation [11].

Similar to Hofer's work, the average exit velocity of each species at angle $\theta$ is:

$$
\left\langle v_{a, i}(\theta)\right\rangle=\sqrt{\frac{2 Z_{i} e V_{a, i}(\theta)}{M_{i}}}
$$

To account for the axially directed thrust power produced, the axial component of ion velocity for each ion species is given in Eq. (20). However, in terms of performance, the average axial accelerating voltage for each ion species is the important variable and 
is given in Eq. (21).

$$
\begin{gathered}
\left\langle v_{z, i}(\theta)\right\rangle=\left\langle v_{a, i}(\theta)\right\rangle \cos (\theta) \\
V_{z, i}(\theta)=M_{i} /\left(2 e Z_{i}\right)\left\langle v_{z, i}(\theta)\right\rangle^{2}=V_{a, i}(\theta) \cos ^{2}(\theta)
\end{gathered}
$$

The current density of all ion species at angle $\theta$ is

$$
\begin{aligned}
j(\theta) & =\sum n_{i}(\theta) q_{i}\left\langle v_{i}(\theta)\right\rangle \\
& =\sqrt{2 / M_{i}} n_{b}(\theta) e^{3 / 2} \sum \zeta_{i}(\theta) Z_{i}^{3 / 2} \sqrt{V_{a, i}(\theta)}
\end{aligned}
$$

The ion mass flux for all species at angle $\theta$ is given in Eq. (23).

$$
\begin{aligned}
& \Gamma_{i}(\theta)=M_{i} \sum n_{i}(\theta)\left\langle v_{i}(\theta)\right\rangle \\
& \quad=n_{b}(\theta) \sqrt{2 e M_{i}} \sum \zeta_{i}(\theta) Z_{i}^{1 / 2} \sqrt{V_{a, i}(\theta)}
\end{aligned}
$$

By solving Eq. (22) for $n_{b}$ and combining it with Eq. (23), the ion mass flux equation reduces to

$$
\Gamma_{i}(\theta)=\frac{M_{i}}{e} j(\theta) \frac{\sum \zeta_{i}(\theta) Z_{i}^{1 / 2} \sqrt{V_{a, i}(\theta)}}{\sum \zeta_{i}(\theta) Z_{i}^{3 / 2} \sqrt{V_{a, i}(\theta)}}
$$

The average acceleration voltage of the different ion species may vary by a few volts. Kim [25] and King [35] both observe that the difference of these voltage potentials is on the order of the ionization potentials [36]. Because of the fact that the RPA measures the energy-to-charge ratio of the ions, the measured most probable kinetic energy is an average acceleration voltage over all species. In addition to this, singly charged ions account for a vast majority of the ion species in the plume. For these reasons, the average acceleration voltage is assumed to be equal for all ion species. A comprehensive discussion of this assumption is covered in Hofer's thesis [11]. With this assumption, Eq. (24) becomes:

$$
\Gamma_{i}(\theta)=\frac{M_{i}}{e} j(\theta) \frac{\sum \zeta_{i}(\theta) Z_{i}^{1 / 2}}{\sum \zeta_{i}(\theta) Z_{i}^{3 / 2}}
$$

The total ion mass flow rate in the beam can be calculated by Eq. (26).

$$
\dot{m}_{b}=2 \pi z^{2} \int_{0}^{\pi / 2} \Gamma_{i}(\theta) \sin (\theta) d \theta
$$

Assuming that the species fraction measurement along the centerline is a good representation of the species fractions in the entire thruster plume, the total ion beam mass flow rate can be given in the much simpler form shown in Eq. (27). Since the large majority of the beam mass flow rate will fall near the centerline of the thruster, this assumption is a good one.

$$
\dot{m}_{b}=\frac{I_{b} M_{i}}{e}\left[\sum \Omega_{i} / Z_{i}\right]_{\mathrm{CL}}
$$

To calculate the average mass-weighted axial acceleration voltage, the mass flux multiplied by the axial acceleration voltage is integrated from 0 to $90 \mathrm{deg}$ [Eq. (28)]. For this analysis, it will be necessary to make one more assumption: the species fractions are constant at different angular positions in the plume. Kim [25] did see species variation at different angular positions off thruster axis. If the multiply charged species are formed in a multistep process (as opposed to a single ionizing collision) they would begin accelerating farther downstream, which would result in a larger divergence angle for multiply charged species. Although Kim found species fraction variation at different angular positions, the previously mentioned assumption will be necessary to proceed further with our analysis. Because such a large majority of the beam is located near the centerline of the thruster in a region of little species fraction variation, the error associated with this assumption will remain small. Additionally, the species fraction effects are of second order, and so this assumption will still yield reliable results. Note that if $E \times B$ data are taken at all angular positions, this assumption would not be needed.

We are now able to solve the voltage utilization efficiency. The new expression for voltage efficiency is given in Eq. (29).

$$
\begin{gathered}
\left\langle\dot{m}_{i}(\theta) V_{z}(\theta)\right\rangle=2 \pi z^{2} \int_{0}^{\pi / 2} \Gamma_{i} V_{z}(\theta) \sin (\theta) d \theta \\
\eta_{v}=\frac{2 \pi z^{2}}{V_{D}\left[\sum \Omega_{i} / Z_{i}\right]_{\mathrm{CL}}} \int_{0}^{\pi / 2} V_{a}(\theta) g(\theta) \frac{\sum \zeta_{i}(\theta) Z_{i}^{1 / 2}}{\sum \zeta_{i}(\theta) Z_{i}^{3 / 2}} \\
\times \cos ^{2}(\theta) \sin (\theta) d \theta \\
g(\theta)=\left[j(\theta) / I_{b}\right]
\end{gathered}
$$

The term $g(\theta)$ is the ion current density term divided by the beam current term, which gives particular advantages to the presented analysis. Faraday probes are well known to have a relatively moderate degree of error and are often only reliable in identifying relative trends. The advantage of dividing the current density by the total beam current measured by the Faraday probe is to remove this magnitude error. With this normalized current density function, the beam can be integrated using only the relative current density change, and the true beam current is left undetermined to be calculated later in the efficiency analysis. For this analysis, it is advantageous to use a Faraday probe that filters charge exchange ions (e.g., an MFFP [27]), because CEX ions contribute a large portion of the beam at large angles off centerline.

In the Hall thruster plume, the voltage for the primary beam ions is constant for a vast majority of the beam. This result is shown in the results section, next. Experimental results show that elastic collision ions and CEX ions become a significant portion of the beam current only outside of the $95 \%$ cone half angle ( $\sim 6 \mathrm{deg}$ off centerline). For this reason, it is safe to assume that the average acceleration voltage is constant at all angular positions in the beam. In fact, the difference in beam efficiency is less than one half of $1 \%$ with this assumption. This assumption further simplifies Eq. (29), which becomes Eq. (31), accordingly. The beam divergence efficiency is given in Eq. (32) and requires only one $E \times B$ measurement on centerline and one Faraday probe sweep.

$$
\eta_{v}=\frac{V_{a}}{V_{D}} \frac{\sum \zeta_{i} Z_{i}^{1 / 2}}{\left[\sum \Omega_{i} / Z_{i}\right] \sum \zeta_{i} Z_{i}^{3 / 2}}\left[2 \pi z^{2} \int_{0}^{\pi / 2} g(\theta) \cos ^{2}(\theta) \sin (\theta) d \theta\right]
$$

$$
\eta_{\mathrm{div}}=\frac{\sum \zeta_{i} Z_{i}^{1 / 2}}{\left[\sum \Omega_{i} / Z_{i}\right] \sum \zeta_{i} Z_{i}^{3 / 2}}\left[2 \pi z^{2} \int_{0}^{\pi / 2} g(\theta) \cos ^{2}(\theta) \sin (\theta) d \theta\right]
$$

The relative error in the accelerating voltage efficiency is calculated from the RPA uncertainty and is equal to $1.6 \%$. The beam divergence efficiency is calculated by analyzing a large number of MFFP measurements and comparing the results to nude Faraday probe data. The variance of the beam divergence efficiency is then calculated and is used to arrive at a conservative estimate for the beam divergence error. The relative error of the beam divergence efficiency is $2.5 \%$. This method is conservative because the nude Faraday probe is well known to vastly overpredict the beam current at large angles off centerline and can be considered the worst case scenario. At last, the relative error of the voltage utilization efficiency is equal to $3 \%$. 


\section{Experimental Results}

\section{A. Operation Points of Interest}

The operation points of interest and performance values for each are given in Table 1. Xenon data are taken at $700 \mathrm{~V}, 6$ and $8 \mathrm{~kW}$ with and without the trim coil. There are two corresponding krypton points for each xenon point. One krypton point matches the volumetric flow rate of the analogous xenon case, and the other matches the power of the xenon case. Krypton propellant would most likely be chosen over xenon for a particular mission because of its superior specific impulse. For this reason, operation points with large discharge voltages are chosen. The choice of $700 \mathrm{~V}$ discharge voltage also has the benefit of minimizing the krypton-xenon efficiency deficit. The krypton efficiency is optimized for high anode flow rates and at high discharge voltages. This finding is expected because previous work $[4,5]$ suggests that the krypton efficiency gap is largely due to deficient propellant utilization efficiency.

\section{B. Retarding Potential Analyzer}

An example of the RPA measurements appear in Fig. 6. As seen by other experimentalists [37], the RPA identifies three species of ions in the energy-per-charge distribution curves: the primary beam ions, ions that have undergone elastic collisions, and CEX ions. Interestingly, within the $90 \%$ beam divergence half angle (found to be approximately $50 \mathrm{deg}$ in the MFFP results), the ions are almost solely primary beam ions and the most probable velocity is roughly constant. Near the $95 \%$ beam divergence half angle $(\sim 6 \mathrm{deg})$ the current collected from ions that have undergone elastic collisions are on the same order as the beam ions. Beyond the $95 \%$ beam divergence half angle, the CEX ions are the dominant ion species.

The most probable voltage is given by the dominant peak in energy-per-charge distribution function. This value is taken to be the average beam voltage (ion kinetic energy in electron volts). It is shown in Fig. 6, that the most probable voltage is constant inside of the 30-deg cone of the Hall thruster plume. For this reason, the average ion kinetic energy inside the 30-deg cone is used in the acceleration efficiency and is given in Table 2. The most probable beam voltage and the full width half maximum (FWHM) of the beam voltage distribution function are also given in the following table.

The most probable voltage is approximately the same for xenon and krypton. One might expect the most probable voltage to be marginally lower for krypton because of its higher ionization potential. However, this effect, if it exists, would be negligible for these operation points. The trim coil does increase the most probable ion voltage by around $1 \%$. The acceleration efficiency is then calculated by simply measuring the average ion voltage (kinetic energy).

The dispersion efficiency characterizes the effect of the spread in ion velocities in the Hall thruster plume [1] [Eq. (33)]. An example of the spread in ion energy-per-charge at $30 \mathrm{deg}$ off centerline is displayed in Fig. 7. Xenon appears to have approximately a $25 \%$ larger FWHM in the ion beam voltage distribution function than krypton. This effect counteracts any voltage loss due to krypton's higher ionization potential. These results suggest that more of the

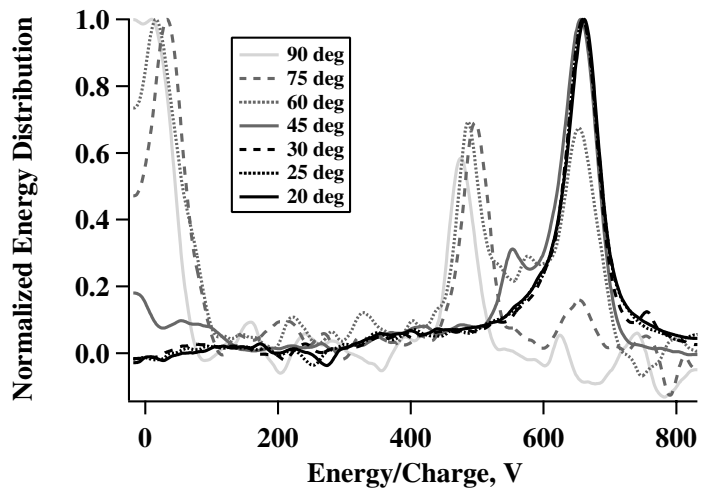

Fig. 6 Voltage distribution functions at different angular locations for operation point 12 .

ionization occurs upstream of the acceleration zone in the krypton case. The trim coil reduces the ion velocity spread by approximately $13 \%$, which leads to improved acceleration. Although the ion velocity spread is important, the dispersion efficiency is difficult to calculate and the average ion voltage is more simply calculated from the most probable voltage.

$$
\eta_{d}=\left\langle v_{a}\right\rangle^{2} /\left\langle v_{a}^{2}\right\rangle
$$

\section{C. $E \times B$ Probe}

The $E \times B$ results are shown in Table 3 . Although $\mathrm{Xe}^{+4}$ is clearly visible in the xenon data sweeps, only charge states up to and including $\mathrm{Kr}^{+3}$ could be resolved for the krypton measurements. Because of higher ionization energies, it is not surprising that krypton displays fewer multiply charged species. Accordingly, the charge utilization is approximately $2 \%$ higher for krypton.

Table 2 RPA Results for the $700 \mathrm{~V}, 6$ and $8 \mathrm{~kW}$ operation points

\begin{tabular}{lcccc}
\hline \hline Point no. & $\begin{array}{c}\text { Most probable } \\
\text { voltage, V }\end{array}$ & $\begin{array}{c}\text { Voltage } \\
\text { loss, V }\end{array}$ & $\begin{array}{c}\text { Voltage spread } \\
\text { FWHM, V }\end{array}$ & $\begin{array}{c}\text { Acceleration } \\
\text { efficiency, \% }\end{array}$ \\
\hline 1 & 652 & 48 & 92.6 & 93.1 \\
2 & 662 & 38 & 79.4 & 94.6 \\
3 & 651 & 49 & 69.0 & 93.0 \\
4 & 664 & 36 & 59.8 & 94.9 \\
5 & 653 & 47 & 68.6 & 93.3 \\
6 & 663 & 37 & 58.4 & 94.7 \\
7 & 656 & 44 & 92.9 & 93.7 \\
8 & 670 & 30 & 87.2 & 95.7 \\
9 & 656 & 44 & 79.0 & 93.7 \\
10 & 654 & 46 & 68.8 & 93.4 \\
11 & 657 & 43 & 68.3 & 93.9 \\
12 & 662 & 38 & 57.6 & 94.6 \\
\hline \hline
\end{tabular}

Table 1 Operating conditions for the $700 \mathrm{~V}, 6$ and $8 \mathrm{~kW}$ cases

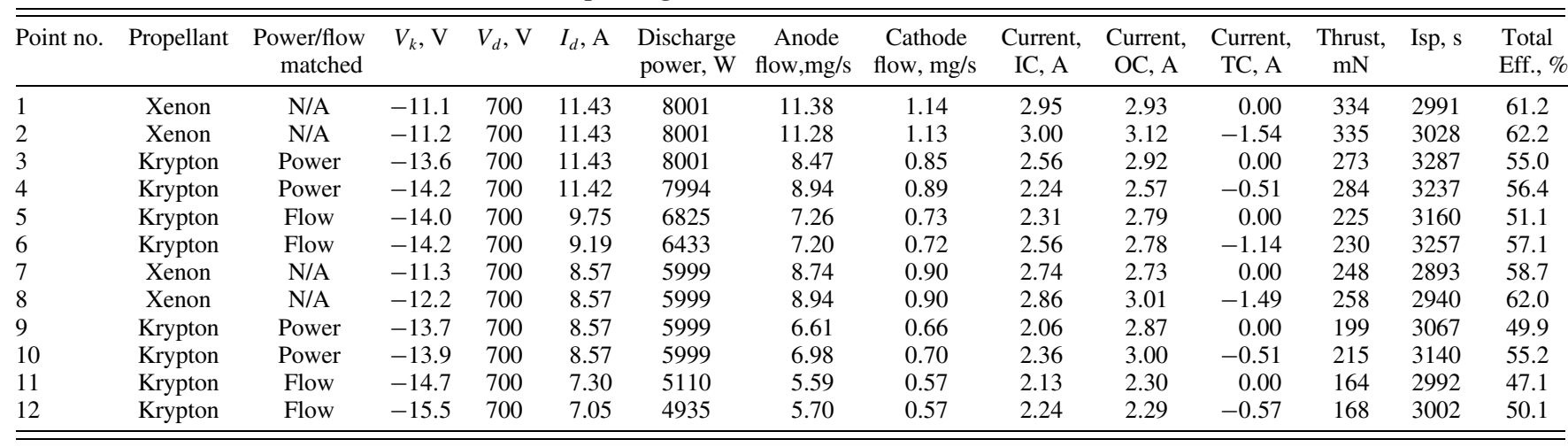




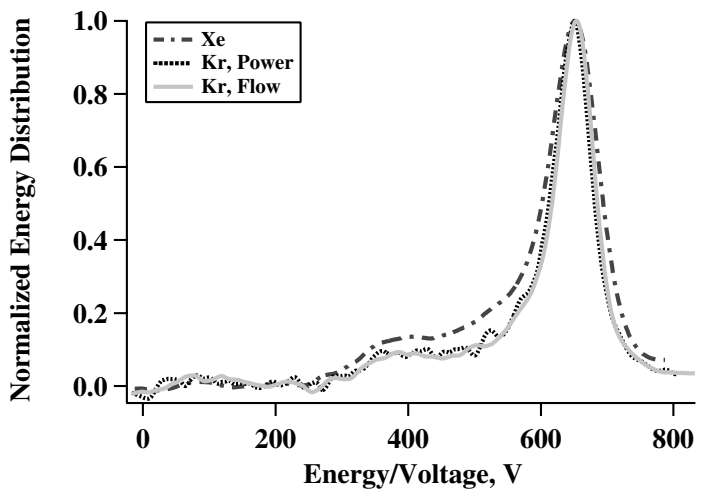

Fig. 7 Voltage distribution comparison at $8 \mathrm{~kW}$ without the trim coil (TC).

\section{Magnetically Filtered Faraday Probe Results}

The beam current and beam divergence half angles from the MFFP results are given in Table 4 . The MFFP data are used in conjunction with $E \times B$ and RPA results to solve the beam divergence efficiency given in Eq. (32). The ion current density for the $8 \mathrm{~kW}$ no-trim-coil data points are given in Fig. 8. The krypton operation points have greater beam divergence half angles than the xenon points ( $\sim 6 \mathrm{deg})$. This trend is consistent with other researchers' findings [6]. The trim coil does not appear to have a significant effect on beam divergence for these experimental conditions. The calculated beam divergence efficiencies are given in the following section.

\section{E. Efficiency Analysis: Current, Propellant, and Beam Divergence Efficiency}

A complete table of the efficiencies for the 6 and $8 \mathrm{~kW}$ operation points is given in Table 5. The propellant utilization and beam divergence appear to be the dominant factors responsible for the efficiency gap between xenon and krypton.

The beam divergence efficiency is between 78 and $89 \%$ for the listed operation points. Xenon has a beam divergence efficiency about $8 \%$ larger than the krypton points. This divergence is a significant contributor to the krypton efficiency gap and results in a voltage utilization efficiency deficit of about $8 \%$.

The propellant utilization for xenon is approximately $90 \%$ and between $80 \%$ and $85 \%$ for krypton. The trim coil appears to have very little effect on propellant utilization for xenon and krypton. The high krypton efficiency results seen with the NASA-457M $[8,9]$ and the NASA-400M [10] experiments are connected to propellant utilization optimization. These thrusters have a large discharge channel that increases krypton residence time and hence the probability of ionization.

Current utilization is approximately the same for the krypton and xenon points. However, the trim coil appears to improve the electron dynamics inside the Hall thruster, which can be seen in the current utilization. The current utilization (shown in Table 5) is between 80 and $89 \%$ for these operation points. The current utilization is
Table 4 Magnetically filtered Faraday probe results for the 6 and $8 \mathrm{~kW}$ operation points

\begin{tabular}{lccc}
\hline \hline Point no. & Beam current, A & 95\% beam div., deg & 90\% beam div., deg \\
\hline 1 & 9.21 & 53.0 & 42.0 \\
2 & 9.33 & 54.5 & 44.5 \\
3 & 8.44 & 60.0 & 49.0 \\
4 & 8.87 & 60.5 & 50.5 \\
5 & 7.57 & 61.5 & 50.5 \\
6 & 7.62 & 60.5 & 50.0 \\
7 & 7.14 & 57.5 & 46.0 \\
8 & 7.20 & 57.5 & 47.0 \\
9 & 6.74 & 63.5 & 52.5 \\
10 & 6.79 & 61.5 & 50.5 \\
11 & 5.56 & 63.5 & 52.5 \\
12 & 5.76 & 62.5 & 51.5 \\
\hline \hline
\end{tabular}

improved by 1 to $6.5 \%$ when the trim coil is in use. This improved current utilization can be explained by the magnetic mirroring effect that, theoretically, is focusing the electrons toward the center of the discharge channel. This effect would reduce electron-wall collisions and near-wall conductivity $[2,38]$.

Several trends can be observed from the tabulated data when plotted vs anode flow rate. As anode flow rate increases, several performance components are improved, including anode efficiency, propellant utilization, current utilization, and beam divergence. Charge utilization is decreased slightly by increased anode flow rate. The acceleration efficiency is not strongly affected.

The anode efficiency is given in Fig. 9. As the anode flow is increased $50 \mathrm{sccm}$, krypton anode efficiency increases by as much as $10 \%$. Xenon performance is largely unaffected by the increased anode flow rate. The finer points of this efficiency improvement are captured in the propellant utilization, charge utilization, and beam divergence efficiency calculations.

Propellant utilization efficiency vs anode flow rate is given in Fig. 10. Propellant utilization is increased by almost $7 \%$ as anode flow rate increases and plateaus to around $86 \%$. This finding is not surprising because a larger anode flow rate will increase the neutral number density and, concurrently, the rate of ionizing collisions. Xenon propellant utilization is approximately constant ( $90 \%)$ for all flow rates. The xenon propellant utilization is already maximized and nothing is gained by increasing anode flow rate. Following the same lines of thought, this trend explains the slight decrease in charge utilization efficiency with increased anode flow rate. That is, the larger neutral number density results in more ionizing collisions and therefore more multiply charged species.

Current utilization efficiency is shown in Fig. 11. For krypton, the current utilization efficiency increases by almost $9 \%$. For the trim coil case, current utilization appears to plateau at around $90 \%$ as flow rate increases. This result may seem counterintuitive because the increasing neutral and plasma density should result in more electronparticle collisions, which should increase the electron cross-field mobility. However, as flow rate increases, ion production increases, which in turn increases the beam current. This result may also

Table $3 E \times B$ results for the $700 \mathrm{~V}, 6$ and $8 \mathrm{~kW}$ operation points

\begin{tabular}{lccccccccc}
\hline \hline Point no. & $\Omega_{1}$ & $\Omega_{2}$ & $\Omega_{3}$ & $\Omega_{4}$ & $\mathrm{Xe}^{+} / \mathrm{Kr}^{+}$ & $\mathrm{Xe}^{+} / \mathrm{Kr}^{+}$ & $\mathrm{Xe}^{3+} / \mathrm{Kr}^{3+}$ & $\mathrm{Xe}^{4+}$ & Charge util. eff., \% \\
\hline 1 & 0.6268 & 0.2219 & 0.1152 & 0.0361 & 0.8832 & 0.0954 & 0.0188 & 0.0026 & 96.02 \\
2 & 0.5938 & 0.1793 & 0.1612 & 0.0657 & 0.8855 & 0.0816 & 0.0279 & 0.0051 & 95.09 \\
3 & 0.6741 & 0.2346 & 0.0913 & 0.0000 & 0.8951 & 0.0910 & 0.0139 & N/A & 96.97 \\
4 & 0.7522 & 0.1818 & 0.0660 & 0.0000 & 0.9254 & 0.0653 & 0.0093 & N/A & 97.60 \\
5 & 0.7161 & 0.2054 & 0.0785 & 0.0000 & 0.9121 & 0.0765 & 0.0114 & N/A & 97.29 \\
6 & 0.7478 & 0.2048 & 0.0474 & 0.0000 & 0.9197 & 0.0736 & 0.0067 & N/A & 97.73 \\
7 & 0.6286 & 0.1956 & 0.1349 & 0.0408 & 0.8903 & 0.0845 & 0.0222 & 0.0030 & 95.79 \\
8 & 0.6565 & 0.1624 & 0.1215 & 0.0597 & 0.9078 & 0.0685 & 0.0195 & 0.0043 & 95.72 \\
9 & 0.8219 & 0.1257 & 0.0525 & 0.0000 & 0.9506 & 0.0425 & 0.0069 & N/A & 98.15 \\
10 & 0.8036 & 0.1362 & 0.0602 & 0.0000 & 0.9451 & 0.0468 & 0.0081 & N/A & 97.96 \\
11 & 0.7840 & 0.1697 & 0.0463 & 0.0000 & 0.9346 & 0.0591 & 0.0063 & N/A & 97.96 \\
12 & 0.7344 & 0.2171 & 0.0485 & 0.0000 & 0.9141 & 0.0790 & 0.0069 & N/A & 97.65 \\
\hline \hline
\end{tabular}




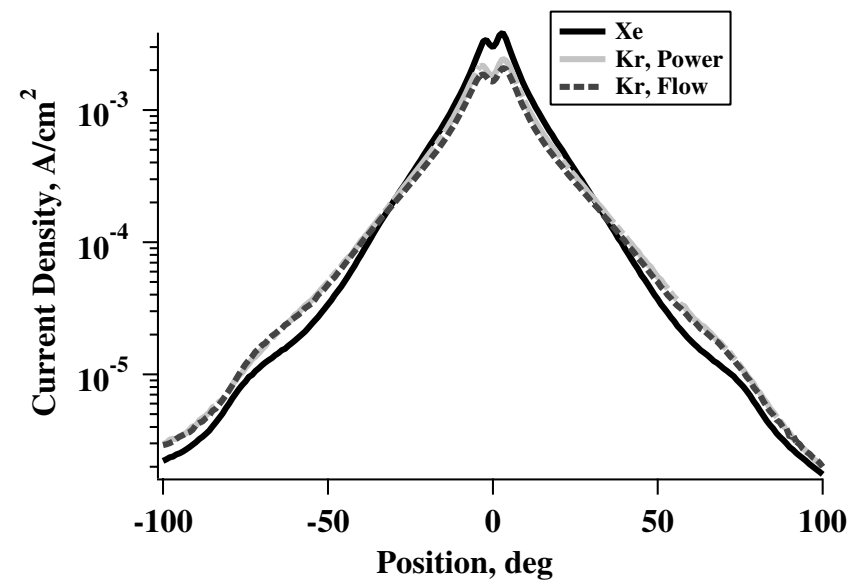

Fig. 8 Ion current density comparison at $8 \mathrm{~kW}$ without the trim coil.

suggest that the dominant mode of axial electron transport is nearwall conductivity. The reason for the improved current utilization is not clear, although the limiting current utilization behavior seen in the krypton trim coil case suggests that there are competing factors at work. Xenon, again, is largely unaffected by the increased flow rate.

Beam divergence efficiency (Fig. 12) is shown to improve slightly for both propellants as anode flow rate is increased. The beam divergence efficiency is improved by about 3 and $4 \%$ for xenon and krypton, respectively. A possible explanation is that as the anode flow rate increases, the ionization rate increases and the ionization zone moves upstream. As the ionization zone is moved farther upstream, ions are able to begin their acceleration earlier in the acceleration zone and more likely in the axial direction.

\section{Conclusion}

An efficiency analysis comparing high-voltage xenon and krypton operation has been conducted. There are a number of efficiency parameters that have been isolated and pinpointed as the causes of the efficiency differences between the two propellants. There have also been a number of trends connected to trim coil operation.

The effects of trim coil operation are as follows:

1) The trim coil proves to have little effect on the overall ionization efficiency. However, the location and dimensions of the ionization zone appear to be affected by the trim coil.

2) The trim coil improves the electron dynamics and voltage utilization.

3) The trim coil improves the anode efficiency by 1 to $6 \%$.

4) The trim coil improves average acceleration voltage $(\sim 1 \%)$.

5) The trim coil improves the ion velocity dispersion efficiency and decreases the FWHM of the ion beam voltage distribution function by $13 \%$.

6) Oddly, the trim coil little effect on beam divergence efficiency.

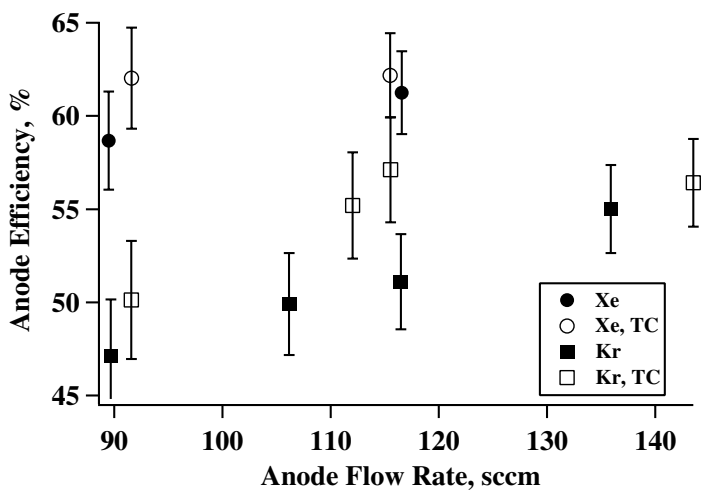

Fig. 9 Efficiency dependence on anode flow rate.

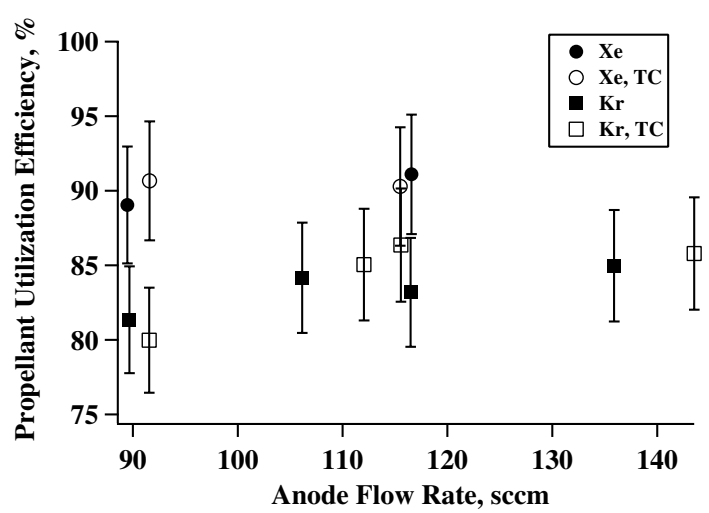

Fig. 10 Propellant utilization efficiency dependence on anode flow rate.

7) The trim coil has little effect on species fractions and propellant utilization efficiency.

8) Current utilization efficiency improves by 1 to $6.5 \%$. The improvement is believed to be connected to the magnetic mirroring effect reducing electron-wall collisions and hence near-wall conductivity.

The differences between krypton and xenon operation are as follows:

1) Total anode efficiency is about 5 to $15 \%$ better for xenon.

2) Krypton has better dispersion efficiency (the FWHM is $25 \%$ higher for xenon) and therefore probably has a larger portion of the ionization occurring upstream of the acceleration zone.

3) Both propellants have the same average ion voltage, resulting in approximately the same acceleration utilization.

4) The beam divergence efficiency is better for xenon (8\%), and, consequently, the voltage utilization is about $8 \%$ better for xenon.

5) Krypton has a better charge utilization efficiency (2\%)

Table 5 The complete efficiency analysis for krypton and xenon operation points

\begin{tabular}{|c|c|c|c|c|c|c|c|c|c|c|}
\hline Point no. & Propell. & $\begin{array}{l}\text { Power/flow } \\
\text { matched }\end{array}$ & $\begin{array}{l}\text { Trim coil } \\
\text { used? }\end{array}$ & $\begin{array}{l}\text { Total anode } \\
\text { eff., } \%\end{array}$ & $\begin{array}{c}\text { Charge util., } \\
\%\end{array}$ & $\begin{array}{l}\text { Acceleration } \\
\text { eff., } \%\end{array}$ & $\begin{array}{l}\text { Divergence } \\
\text { eff., } \%\end{array}$ & $\begin{array}{l}\text { Voltage util. } \\
\text { eff., } \%\end{array}$ & $\begin{array}{l}\text { Current util. } \\
\text { eff., } \%\end{array}$ & $\begin{array}{l}\text { Propellant util. } \\
\text { eff., } \%\end{array}$ \\
\hline 1 & $\mathrm{Xe}$ & N/A & No & 61.2 & 96.0 & 93.1 & 89.2 & 83.1 & 84.3 & 91.1 \\
\hline 2 & $\mathrm{Xe}$ & N/A & Yes & 62.2 & 95.1 & 94.6 & 88.8 & 84.0 & 86.2 & 90.3 \\
\hline 4 & $\mathrm{Kr}$ & Power & Yes & 56.4 & 97.6 & 94.9 & 80.1 & 76.0 & 88.7 & 85.8 \\
\hline 5 & $\mathrm{Kr}$ & Flow & No & 51.1 & 97.3 & 93.3 & 80.8 & 75.4 & 83.7 & 83.2 \\
\hline 6 & $\mathrm{Kr}$ & Flow & Yes & 57.1 & 97.7 & 94.7 & 80.1 & 80.1 & 89.3 & 86.4 \\
\hline 7 & $\mathrm{Xe}$ & N/A & No & 58.7 & 95.8 & 93.7 & 86.6 & 81.2 & 84.7 & 89.1 \\
\hline 10 & $\mathrm{Kr}$ & Power & Yes & 55.2 & 98.0 & 93.4 & 79.9 & 74.6 & 88.8 & 85.1 \\
\hline 11 & $\mathrm{Kr}$ & Flow & No & 47.1 & 98.0 & 93.9 & 78.3 & 73.5 & 80.4 & 81.3 \\
\hline 12 & $\mathrm{Kr}$ & Flow & Yes & 50.1 & 97.6 & 94.6 & 78.9 & 74.6 & 86.1 & 80.0 \\
\hline
\end{tabular}




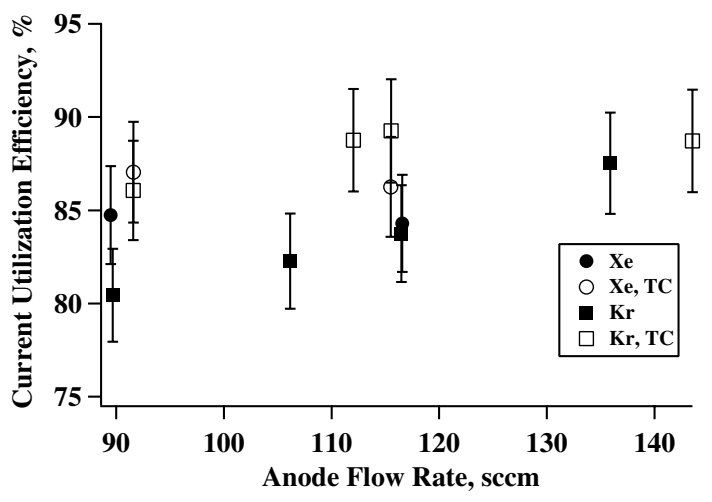

Fig. 11 Current utilization efficiency dependence on anode flow rate.

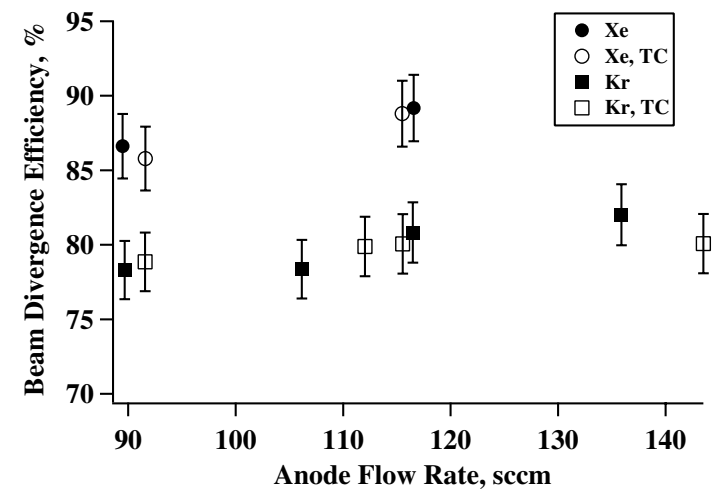

Fig. 12 Beam divergence efficiency dependence on anode flow rate.

6) Both propellants have about the same current utilization efficiency.

7) Propellant utilization is about 5 to $10 \%$ better for xenon. Propellant utilization reaches a maximum of around 90 and $86 \%$ for xenon and krypton, respectively.

The effects of increased anode flow rate are as follows:

1) Increased anode flow rate improves krypton's anode efficiency by as much as $10 \%$.

2) The increased anode flow rate increases the ionization rate. The increased ionization rate improves propellant utilization and, to a lesser degree, hurts charge utilization efficiency.

3) Increased anode flow rate is shown to improve current utilization efficiency.

4) Beam divergence is also slightly improved with increased anode flow rate. The higher number density may move the ionization zone upstream and allow ions to start accelerating earlier in the acceleration zone, which reduces the divergence.

\section{Suggestions for Krypton Design Improvements and Future Work}

Several things can be done to improve the performance of Hall thruster operation with krypton. A longer discharge channel will lead to longer propellant residence times, which will improve the propellant utilization. Higher discharge voltages will increase electron temperatures and ionization collision cross sections, which will increase the probability of an ionizing electron-atom collision. High channel neutral density will also increase the collision rate, which can further increase the propellant utilization.

There should be a focus on improving understanding of krypton's large divergence angle. This is probably related to the internal plasma potential structure and the magnetic field. Future work should focus on the studying the internal potential structure and better understanding the optimum magnetic field structure for krypton propellant.

\section{Acknowledgments}

The authors would like to thank the Association Francois-Xavier Bagnoud for their financial support during Jesse Linnell's graduate studies and the NASA Glenn Research Center for financial support through research grant NCC04GA38G (grant monitor David Jacobson) and for the use of government-furnished equipment. We also thank Joshua L. Rovey for his assistance with data acquisition. Finally, we would like to thank James Haas of the Air Force Research Laboratory, Edwards Air Force Base, CA for design of PEPL's RPA.

\section{References}

[1] Kim, V., "Main Physical Features and Processes Determining the Performance of Stationary Plasma Thrusters," Journal of Propulsion and Power, Vol. 14, No. 5, 1998, pp. 736-743.

[2] Zhurin, V. V., Kaufman, H. R., and Robinson, R. S., "Physics of Closed Drift Thrusters," Plasma Sources Science and Technology, Vol. 8, No. 1, 1999, pp. R1-R20.

[3] Marrese, C., Haas, J. M., Domonkos, M. T., Gallimore, A. D., Tverdokhlebov, S., and Garner, C. E., "The D-100 Performance and Plume Characterization of Krypton," AIAA Paper 96-2969, 1996.

[4] Kim, V., Popov, G., Kozlov, V., Skrylnikov, A., and Grdlichko, D., "Investigation of SPT Performance and Particularities of Its Operation with Krypton and Xenon Mixtures," Electric Rocket Propulsion Society, Paper 2001-065, Cleveland, OH, 2001.

[5] Semenkin, A. V., and Gorshkov, G. O., "Study of Anode Layer Thruster Operation with Gas Mixtures," Electric Rocket Propulsion Society, Paper 1995-078, Cleveland, OH, 1995.

[6] Bugrova, A. I., Lipatov, A. S., Morozov, A. I., and Solomatina, L. V., "Global Characteristics of an ATON Stationary Plasma Thruster Operating with Krypton and Xenon," Plasma Physics Reports, Vol. 28, No. 12, 2002, pp. 1032-1037.

[7] Bugrova, A. I., Lipatov, A. S., Morozov, A. I., and Churbanov, D. V., "On a Similarity Criterion for Plasma Accelerators of the Stationary Plasma Thruster Type," Technical Physics Letters, Vol. 28, No. 10, 2002, pp. 821-823.

[8] Manzella, D. H., Jankovsky, R., and Hofer, R. R., "Laboratory Model 50 kW Hall Thruster," AIAA Paper 2002-3676, 2002.

[9] Jacobson, D. T., and Manzella, D. H., "50 KW Class Krypton Hall Thruster Performance," AIAA Paper 2002-4550, 2002.

[10] Peterson, P. Y., Jacobson, D. T., Manzella, D. H., and John, J. W., "The Performance and Wear Characterization of a High-Power High-Isp NASA Hall Thruster," AIAA Paper 2005-4243, 2005.

[11] Hofer, R. R., "Development and Characterization of High-Efficiency, High-Specific Impulse Xenon Hall Thrusters," Doctoral Thesis, Department of Aerospace Engineering, Univ. of Michigan, Ann Arbor, MI, 2004.

[12] Dushman, S., Scientific Foundations of Vacuum Technique, Vol. 4 Wiley, New York, 1958.

[13] Lide, D. R., CRC Handbook of Chemistry and Physics, 73rd ed., CRC Press, Boca Raton, FL, 1992.

[14] Dymond, J. H., and Smith, E. B., The Virial Coefficients of Pure Gases and Mixtures, a Critical Compilation, Oxford Univ. Press, New York, 1980.

[15] Hofer, R. R., and Gallimore, A. D., "The Role of Magnetic Field Topography in Improving the Performance of High-Voltage Hall Thrusters," AIAA Paper 2002-4111, 2002.

[16] Crow, E. L., Davis, F. A., and Maxfield, M. W., Statistics Manual, Dover, New York, 1960.

[17] Hofer, R. R., and Gallimore, A. D., "Efficiency Analysis of a HighSpecific Impulse Hall Thruster," AIAA Paper 2004-3602, 2004.

[18] Beal, B. E., and Gallimore, A. D., "Energy Analysis of a Hall Thruster Cluster," Electric Rocket Propulsion Society, Paper IEPC-2003-035, Cleveland, OH, 2003.

[19] Hutchinson, I. H., Principles of Plasma Diagnostics, 2nd ed., Cambridge Univ. Press, New York, 1990.

[20] Smith, T. B., Ngom, B. B., Linnell, J. A., and Gallimore, A. D., "Diode Laser-Induced Fluorescence of Xenon Ion Velocity Distributions," AIAA Paper 2005-4406, 2005.

[21] Gulczinski, F. S., "Examination of the Structure and Evolution of Ion Energy Properties of a $5 \mathrm{~kW}$ Class Laboratory Hall Effect Thruster at Various Operational Conditions," Doctoral Thesis, Department of Aerospace Engineering, Univ. of Michigan, Ann Arbor, MI, 1999.

[22] Walker, M. L. R., "Effects of Facility Backpressure on the Performance and Plume of a Hall Thruster," Ph.D. Thesis, Department of Aerospace Engineering, Univ. of Michigan, Ann Arbor, MI, 2005.

[23] Reinsche, C. H., "Smoothing by Spline Functions," Numerische 
Mathematik, Vol. 10, No. 3, 1967, pp. 177-183.

[24] Beal, B. E., "Clustering of Hall Effect Thrusters for High-Power Electric Propulsion Applications," Doctoral Thesis, Department of Aerospace Engineering, Univ. of Michigan, Ann Arbor, MI, 2004.

[25] Kim, S. W., "Experimental Investigations of Plasma Parameters and Species-Dependent Ion Energy Distribution in the Plasma Exhaust Plume of a Hall Thruster," Ph.D. Thesis, Department of Aerospace Engineering, University of Michigan, Ann Arbor, 1999.

[26] Hagstrum, H. D., "Auger Ejection of Electrons from Tungsten by Noble Gas Ions," Physical Review, Vol. 96, No. 2, 1954, pp. 325-335.

[27] Rovey, J. L., Walker, M. L. R., Gallimore, A. D., and Peterson, P. Y., "Evaluation of a Magnetically-Filtered Faraday Probe for Measuring the Ion Current Density Profile of a Hall Thruster," AIAA Paper 20043948, 2004.

[28] Rovey, J. L., "Status of Faraday Cup Magnetic Filter Design," Memorandum, Univ. of Michigan, Ann Arbor, MI, 2002.

[29] Hofer, R. R., Walker, M. L. R., and Gallimore, A. D., "A Comparison of Nude and Collimated Faraday Probes for Use with Hall Thrusters," Electric Rocket Propulsion Society, Paper IEPC-2001-020, Cleveland, $\mathrm{OH}, 2001$.

[30] Brown, S. C., Basic Data of Plasma Physics, American Institute of Physics, New York, 1994.

[31] Sovey, J. S., and Patterson, M. J., "Ion Beam Sputtering in Electric Propulsion Facilities," AIAA Paper 1991-2117, 1991.

[32] Sakabe, S., and Izawa, Y., "Cross Sections for Resonant Charge
Transfers Between Atoms and Their Positive Ions: Collision Velocity < 1 a.u.," Atomic Data and Nuclear Data Tables, Vol. 49, No. 2, 1991, pp. 257-314.

[33] Boyd, I. D., and Dressler, R. A., "Far Field Modeling of the Plasma Plume of a Hall Thruster," Journal of Applied Physics, Vol. 92, No. 4 2002, pp. 1764-1774.

[34] Manzella, D. H., "Hall Thruster Ion Beam Characterization," AIAA Paper 1995-2927, 1995.

[35] King, L. B., "Transport-Property and Mass Spectral Measurements in the Plasma Exhaust Plume of a Hall-Effect Space Propulsion System," $\mathrm{Ph} . \mathrm{D}$. Thesis, Department of Aerospace Engineering, Univ. of Michigan, Ann Arbor, 1998.

[36] Arkhipov, B. A., Bober, A. S., Gnizdor, R. Y., Kozubsky, K. N., Korakin, A. I., Maslennikov, N. A., and Pridannikov, S. Y., "The Results of 7000-Hour SPT-100 Life Testing," Electric Rocket Propulsion Society, Paper IEPC-95-39, Cleveland, OH, 1995.

[37] Mikellides, I., Katz, I., Kuharski, R. A., and Mandell, M. J., "Elastic Scattering of Ions in Electrostatic Thruster Plumes," Journal of Propulsion and Power, Vol. 21, No. 1, 2005, pp. 111-118.

[38] Morozov, A. I., and Savel'ev, V. V., "Theory of the Near-Wall Conductivity," Plasma Physics Reports, Vol. 27, No. 7, 2001, pp. 570.

E. Choueiri Associate Editor 